\title{
РАЗРАБОТКА АВТОМАТИЗИРОВАННОГО МОДУЛЯ ПОСТРОЕНИЯ КОНТРОЛЬНЫХ КАРТ УПРАВЛЯЕМОСТИ ПРОЦЕССОВ НА БАЗЕ ПРОГРАММНОГО ПАКЕТА МАТLАВ
}

\section{DEVELOPMENT OF AN AUTOMATED MODULE FOR CONSTRUCTING CONTROL CHARTS PROCESS BASED ON THE MATLAB SOFTWARE PACKAGE}

M. Tikhonov M. Akulenok O. Shikula

Summary. The article describes an analysis and an example of the implementation of software for constructing process controllability control charts based on the functionality of the MATLAB software package. The existing main software products for supporting statistical control are analyzed: SAP (System Analysis and Program Development System Analysis and Program Development (by the name of the developer company)), 1C, Statistica, MATLAB, Minitab, EMI systems. The mathematical apparatus of control charts is presented, as well as the results of approbation of the developed software module.

Keywords: process control, control charts, software, MATLAB, statistical process control.

\author{
Тихонов Мартин Робертович \\ К.т.н., дочент, Национальный исследовательский \\ университет «МИЭТ» \\ kurotenshi91@yandex.ru \\ Акуленок Марина Викторовна \\ К.т.н., дочент, Национальный исследовательский \\ университет «МИЭТ» \\ amv@s2q.ru \\ Шикула Ольга Сергеевна \\ Старший преподаватель, Национальный \\ исследовательский университет «МИЭТ» \\ oshik78@mail.ru
}

Аннотация. В статье приведен анализ и пример реализации программного обеспечения построения контрольных карт управляемости процессов на основе функционала пакета программных средств MATLAB. Проанализированы существующие основные программные продукты поддержки статистического контроля: SAP (System Analysis and Program Development Системный анализ и разработка программ (по наименованию компании-разработчика)), 1C, Statistica, MATLAB, Minitab, EMI-системы. Представлен математический аппарат контрольных карт, а также результаты апробации разработанного программного модуля.

Ключевые слова: управление процессами, контрольные карты, программное обеспечение, MATLAB, статистическое управление процессами.

сти, позволяет решать и даже предупреждать проблемы, связанных с изменчивостью.

ко известный инструмент управления качествол

Они применяются для обеспечения уверенности в том, что производимая продукция обладает требуемым качеством [1]. При рациональном применении статистических методов управления качеством предприятия приобретают возможность системного подхода к анализу процессов и выработке управленческих решений, направленных на повышение качества продукции, т.е. получают возможность реализовать такие принципы международной серии стандартов ISO 9000, как «принятие решений, основанное на фактах» и «постоянное совершенствование» $[1,2]$.

Статистические методы помогают измерить (оценить), описать, проанализировать, а также смоделировать изменчивость, в том числе при наличии ограниченного объема данных. Статистический анализ данных может способствовать лучшему пониманию природы, сроков и факторов, влияющих на причины изменчиво-
Статистическое управление процессами (statistical process control - SPC) на основе анализа обычных (случайных) и особых (неслучайных) причин изменчивости [3], дает возможность не только выявить отклонения от установленных требований, но и применять полученную информацию с целью управления и совершенствования процессов, в частности для улучшения их показателей и предупреждения появления брака [3].

Однако на практике применение статистического управления процессами ограничивается: неподготовленностью персонала, трудоемкостью, особенно при попытках работать с инструментами SPC вручную, потерями времени на принятие решений.

Досадными промахами в некоторых технологических исследованиях является принятие решений 


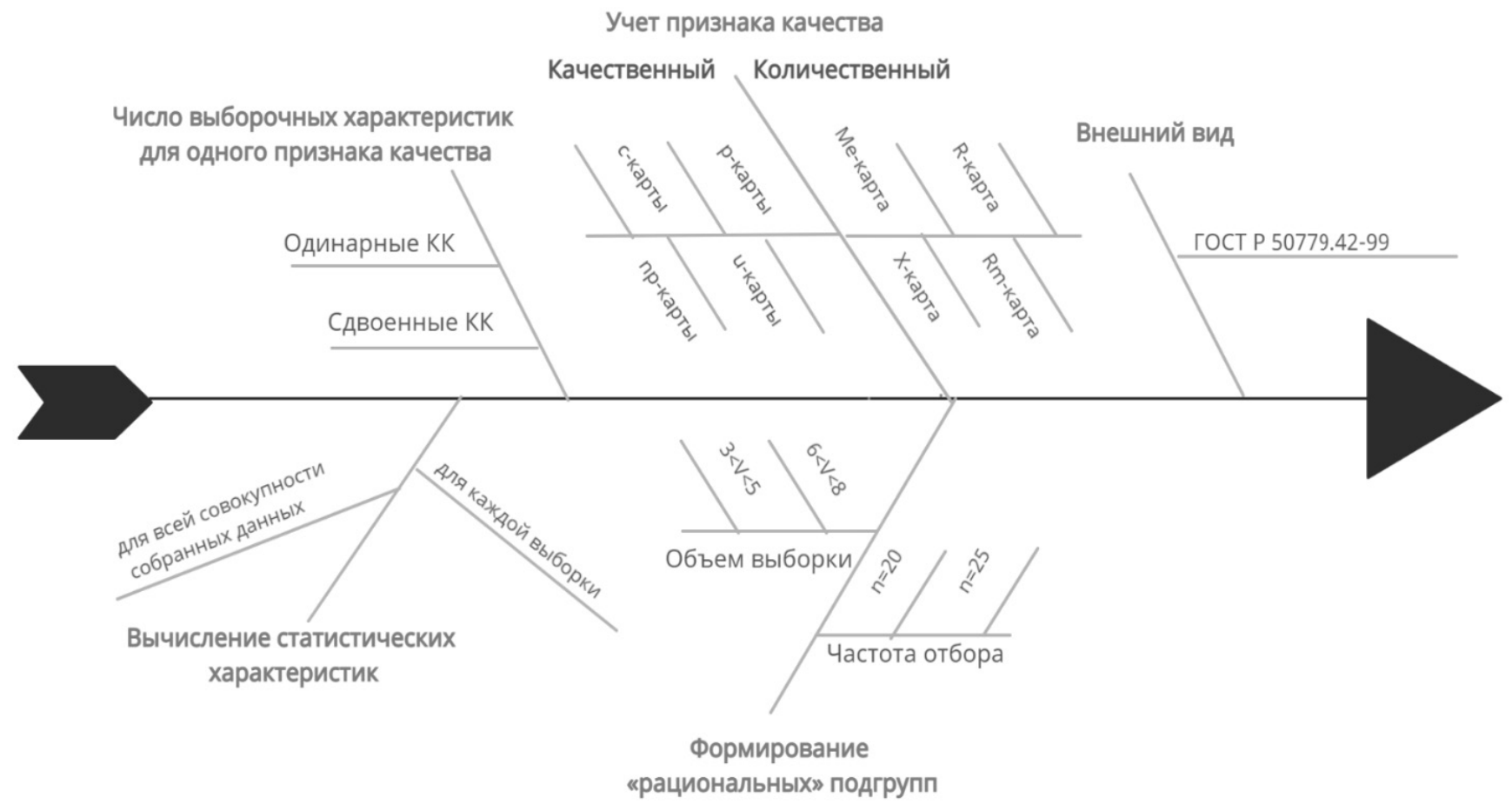

Рис. 1. Результаты анализа «Факторы, определяющие структуру ПО для работы с КК»

в отношении статистически неуправляемого процесса. Неспособность отличить действие особых причин вариаций от действия обычных (общих) причин и отличить устойчивый процесс от нестабильного процесса (так называемые ошибки первого и второго рода), приводит к росту дефектности продукции и к отрицательным экономическим последствиям, затратам, возникающим либо у поставщика, либо у потребителя. Понимание причинно-следственных отношений между параметрами готовой продукции, параметрами незавершенной продукции и параметрами процесса позволяет минимизировать отклонения в качестве готовой продукции [3].

Главным инструментом, используемым в SPC, считаются контрольные карты (КК) управляемости процессов (или контрольные карты Шухарта). Они обеспечивают возможность определить показатели процесса, свидетельствующие о состоянии процесса, его статистической управляемости или неуправляемости и разработать стратегию по улучшению его качества [3].

Специалист, применяющий КК, тратит значительную часть времени на ручное построение КК и расчет основных характеристик процесса. Для того, чтобы увеличить время, на анализ КК, подготовку и принятие решений, необходимо автоматизировать все рутинные операции процесса, например разработку (подготовку) шаблона и построение самих КК. Инструментом автоматизации построения КК может служить специализированное программное обеспечение (ПО) или настроенный модуль информационной системы.

Основные требования, определяющие структуру ПО (представлены на рисунке 1), а именно:

- требования к функционалу, нормативно-методической документации, к техническому обеспечению;

- требования к пользовательскому интерфейсу контрольных карт в программе:

- желаемый дизайн бланка;

- учет признака качества (качественный или количественый тип);

- число выборочных характеристик (одинарные или сдвоенные);

- вычисление статистических характеристик (для всей совокупности собранных данных или для каждой выборки);

- формирование «рациональных» подгрупп (выбор объема подгруппы и частоты взятия выборки).

Часто используемыми информационными системами для поддержки статистического контроля являются: SAP (System Analysis and Program Development - Системный анализ и разработка программ (по наименованию компании-разработчика)), 1C, Statistica, MATLAB, Minitab, ЕMI-системы (Enterprise Manufacturing Intelligence - системы производственной отчетности и аналитики) [4]. 
Таблица 1. Сравнение информационных систем

\begin{tabular}{|c|c|c|c|c|c|}
\hline \multirow{2}{*}{$\begin{array}{c}\text { Критерий } \\
\text { сравнения } \\
\text { систем }\end{array}$} & \multicolumn{5}{|c|}{ Название системы } \\
\cline { 2 - 6 } & SAP & $\mathbf{1 C}$ & Statistica & Matlab/Minitab & EMI \\
\hline $\begin{array}{c}\text { Стоимость } \\
\text { внедрения }\end{array}$ & $>1$ млн. & $\begin{array}{c}>100 \\
\text { тыс. }\end{array}$ & 20 тыс./год & 70 тыс./год & $>3$ млн. \\
\hline ЕSВ & отдельно & нет & нет & нет & есть \\
\hline $\begin{array}{c}\text { Доступность } \\
\text { исходного кода }\end{array}$ & нет & да & нет & нет & нет \\
\hline
\end{tabular}

Сравнительный анализ перечисленных программных продуктов проведен с использованием следующих критериев: стоимость внедрения, интеграционная шина и доступность исходного кода.

Стоимость приобретения и внедрения программного обеспечения - существенная статья расходов для предприятия. Можно выделить две основные статьи затрат: на основной продукт (лицензии) и на вспомогательное ПО (операционные системы для серверов, приложения для сотрудников, защиты информации и др.).

Интеграционная шина (ESB - Enterprise Service Bus) это программно-аппаратное решение, которое позволяет объединить существующие приложения и источники данных. Интеграция обеспечивает единый интерфейс взаимодействия с приложениями и данными. Каждое приложение (информационная система) подключается только к ESB. Интеграционная шина выполняет следующие функции: маршрутизация сообщений между различными информационными системами и гарантия доставки и целостности сообщений. При этом должна быть реализована обработка отказов системы. Даже если информационная система не работала в момент формирования сообщения, по возвращению в работоспособное состояние информация должна быть обработана.

Открытый код способствует адаптации системы под специфические нужды предприятия. Доступность открытого кода также позволяет усовершенствовать существующую систему вместо приобретения новой, однако вводит дополнительные требования к квалификации оператора.

Результаты сравнения информационных систем приведены в Таблице 1.

Наибольшей популярностью среди предприятий РФ пользуются системы 1С, международных предприя- тий - SAP, транснациональных корпораций - EMI-системы. Научно-исследовательские центры некоторых предприятий используют Statistica и Minitab. Отдельного рассмотрения требует вопрос о модернизации существующих информационных систем на предприятии (вместо закупки новых) [4].

Также распространенным инструментом для решения этого рода задач является Microsoft Excel — это программа, которая входит в состав Microsoft Office. Она позволяет работать с электронными таблицами и строить различные диаграммы. Стоит отметить, что при таком функционале менеджеру по качеству приходится вручную формировать новую форму для фиксирования данных, чтобы построить контрольную карту конкретного типа - это кропотливый и длительный процесс.

Таким образом сложно найти универсальный программный продукт, который решал бы вышеперечисленные требования. Нецелесообразно приобретать дорогостоящий программный продукт (стоимость такого программного продукта от 20000 в год и выше), если в нем использовать только какую-то часть, а остальное останется непригодным. Поэтому желательно иметь программу, реализующую необходимую группу задач. Разработке такого программного обеспечения посвящена данная работа.

Первым этапом разработки программного обеспечения являлся выбор языка или платформы реализации. Для ПО, ориентированного на построение диаграмм на основе математических формул и предназначенного для статистического контроля процессов, оптимальным вариантом является платформа с вложенной подсистемой автоматического построения диаграммы. Выбор был ориентирован на широко распространенную платформу - MATLAB [5]. В ней могут быть реализованы специальные модули, выполняющие статистический контроль и построение контрольных карт. 


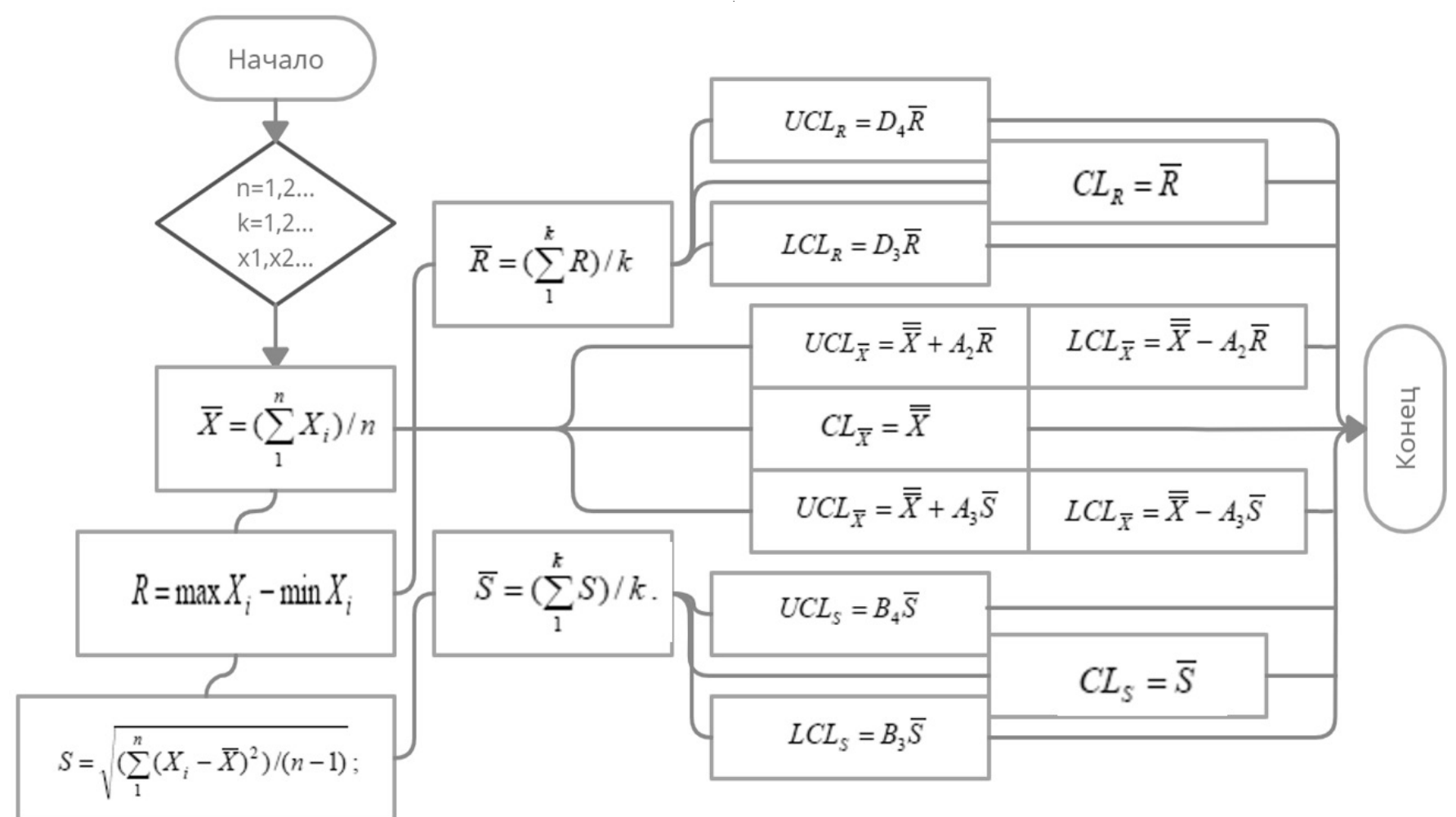

Рис. 2. Схема обобщенного алгоритма построения пар $\overline{\mathrm{X}}-\mathrm{R}$ карты и $\overline{\mathrm{X}}$-S карты

Разработанное приложение позволяет на данном этапе строить три типа контрольных карт: контрольные

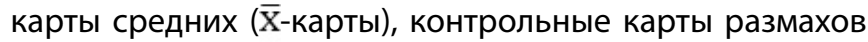
(R-карты), контрольные карты среднеквадратических отклонений (s-карты) и их комбинации ( $\overline{\mathrm{X}}-\mathrm{R}-$ и $\overline{\mathrm{X}}$ - -s карты). Выбор типа карты зависит как от особенностей анализируемого процесса, так и от поставленных целей и задач. Ниже приведен алгоритм работы с созданным ПО, включающий в себя главные характеристики, формулы и коэффициенты для построения контрольных карт, которые были применены в разрабатываемом программном обеспечении. Шаблон контрольных карт, формулы, коэффициенты, используемые в приложении - приведены в соответствии с ГОСТ Р ИСО 7870-2-2015 [6].

Порядок работы с модулем:

- ввести первоначальные данные:

- $\mathrm{n}$ - число индивидуальных значений в подгруппе или объем подгруппы,

- $\mathrm{k}$ - число подгрупп, использованных для вычисления контрольных границ,

- $\mathrm{xi}$ - таблица значений исследуемой(измеренной) характеристики качества;

- определиться с типом карт, которые будут строиться, формулы, коэффициенты выбираются автоматически. Создан модуль соответствующих коэффициентов, программа обращается к этим коэффициентам в зависимости от типа карты и объема подгруппы.

- нажать кнопку «построить».

Схема обобщенного алгоритма построения пар $\overline{\mathrm{X}}-\mathrm{R}$ карты и $\overline{\mathrm{X}}$-s карты представлена на рисунке 2.

Главное окно разработанного приложения представляет собой форму бланка для ведения контрольной карты. Данный программный продукт функционирует на базе операционной системы Windows. Интерфейс программы представлен на рисунке 3.

Апробация разработанного приложения Maps проходила в два этапа:

- тестирование демо-версии;

- апробация «рабочей версии» приложения.

Тестирование демо-версии проводилось с группой экспертов, уверенно-владеющих ручным способом ведения и анализа контрольных карт. На этом этапе был оптимизирован пользовательский интерфейс приложения, даны рекомендации разработчикам, например, первоначально построенная КК стиралась в момент начала нового сеанса работы, поэтому впоследствии предусмотрели сохранение построенных контрольных карт. 

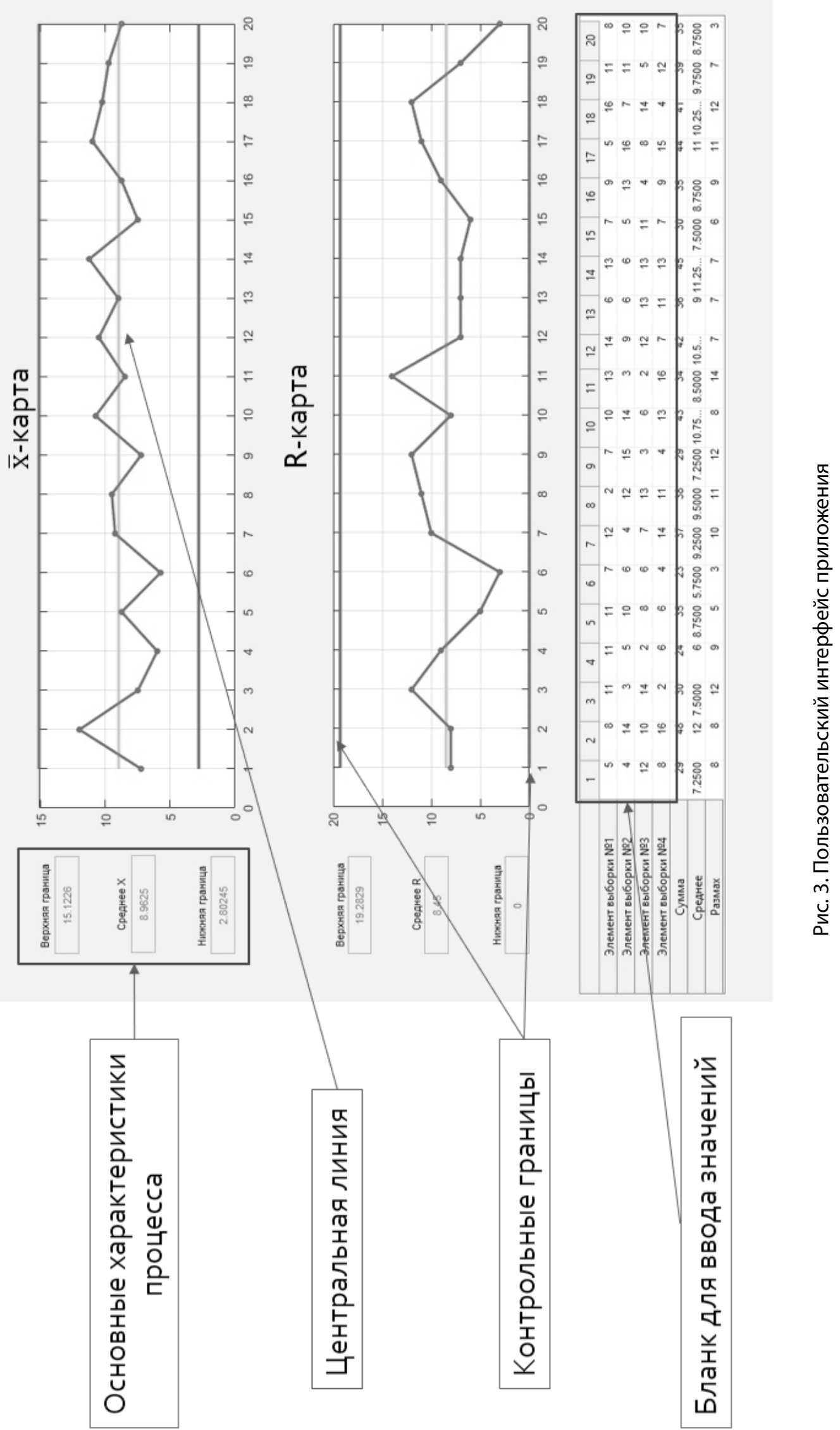

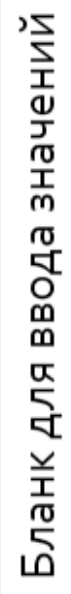


На втором этапе апробация проводилась с двумя группами экспертов:

у уверенно владеющие КK;

- применяющие впервые.

Общий охват экспертов, участвовавших в апробации, составил 13 человек.

Апробация преследовала следующую цель - настроить приложение на запросы потребителей: сделать интуитивно-понятный интерфейс и обеспечить прослеживаемость расчетов, необходимых для построения КK.

Проблем с навигацией и логикой выполнения задач не возникло ни в одной из групп экспертов, поскольку была разработана инструкция по установке и применению, что подтвердило качество разработанной инструкции.

В ходе апробации с первой группой было выявлен ряд достоинств использования средств автоматизации для построения КК. По большей части это связано с исключением человеческого фактора: уменьшением времени на расчет основных характеристик, заполнением шаблона контрольного листа и построением диаграмм. Также во время проведения апробации было выявлено следующее преимущество - возможность выбора типа пары KK ( $\overline{\mathrm{X}}-\mathrm{R}$ карты или $\overline{\mathrm{X}}-\mathrm{S}$ карты) в приложении способствует выявлению чувствительности используемой пары, что позволяет подобрать наиболее оптимальную комбинацию КК для каждого процесса.

В целом, апробация показала, что представление работы с КК в автоматизированном виде позволяет новичкам в три раза быстрее овладеть навыками работы с КК, а уверенным пользователям - на $30 \%$ больше уделить время анализу KK.

Эксперты первой группы оставили пожелание:

- внедрить в программу автоматическое заполнение ячеек таблицы путем «вставки» скопированных значений, которое помогло бы ускорить введение значений в ячейки;

- сместить таблицу с заданными значениями параллельно диаграммам контрольных карт в соответствии с номером выборки. Это в перспективе сократит количество ошибок при чтении КК аналитиком.

При решении исследовательских задач в области технологических процессов сотрудники лабораторий вузов зачастую не учитывают находится ли процесс в состоянии статистической устойчивости, это может приводить к ложным выводам, поскольку корреляция между факторами, влияющими на процесс, может искажаться особыми причинами изменчивости [3]. Разработанное приложение Марs поможет идентифицировать состояние процесса и исключить ошибочные выводы.

\section{ЛИТЕРАТУРА}

1. ГОСТ Р ИСО 9001-2015 Системы менеджмента качества. Требования = Quality management systems. Requirements: Национальный стандарт РФ.Введ. 01.11.2015.- М.: Стандартинформ, 2015.

2. ГОСТ Р ИСО 9000-2015 Системы менеджмента качества. Основные положения и словарь = Quality management systems. Fundamentals and vocabulary: Haциональный стандарт. - Введ. 01.11.2015.— - М.: Стандартинформ, 2015.— [88 л.].— URL: http://docs.cntd.ru/document/1200124393/ (дата 0бращения 19.04.2020)

3. Акуленок М.В., Шикула 0.С. Учебное пособие. Статистическое управление процессами. Часть 1. Контрольные карты управляемости. М.: РИ0 МИЭТ, 2013, стр. 84

4. Локтев Д.А. Статистическое управление производственными процессами - ключ к успеху современного промышленного предприятия Текст научной статьи по специальности «Прочие технологии» Локтев Д.А. https://cyberleninka.ru/article/n/statisticheskoe-upravlenie-protsessami-kak-sposob-snizheniyaproizvodstvennyh-zatrat-i-povysheniya-kachestva-produktsii/viewer

5. Корнеев, В.И. Визуализация в научных исследованиях: учебное пособие / В.И. Корнеев, Л.Г. Гагарина, М.В. Корнеева.— Москва: Инфра-М, 2021.— 400 c.

6. ГОСТ Р ИСО 7870-2-2015 Статистические методы контрольные карты Часть 2 Контрольные карты Шухарта statistical methods. Control charts. Part 2. Shewhart control charts

7. Акуленок М.В., Шикула 0.С., Шикула Р.Д. Алгоритмизация анализа стабильности производственных процессов //в Сборнике: Актуальные проблемы информатизации в цифровой экономике и научных исследованиях. материалы Международной научно-практической конференции. 2019. С. 5-10

(c) Тихонов Мартин Робертович (kurotenshi91@yandex.ru),

Акуленок Марина Викторовна ( amv@s2q.ru ), Шикула Ольга Сергеевна ( oshik78@mail.ru ).

Журнал «Современная наука: актуальные проблемы теории и практики» 